BMJ Open Diabetes

Research \& Care

\section{Does the effect of lifestyle intervention for individuals with diabetes vary by food insecurity status? A preplanned subgroup analysis of the REAL HEALTH randomized clinical trial}

To cite: Berkowitz SA, Chang Y, Porneala B, et al. Does the effect of lifestyle intervention for individuals with diabetes vary by food insecurity status? A preplanned subgroup analysis of the REAL HEALTH randomized clinical trial. BMJ Open Diab Res Care 2020;8:e001514. doi:10.1136/ bmjdrc-2020-001514

- Additional material is published online only. To view, please visit the journal online (http://dx.doi.org/10.1136/ bmjdrc-2020-001514).

An earlier version of these findings was presented as a poster at the American Diabetes Association Scientific Sessions, June 8, 2019, in San Francisco, California.

Received 27 April 2020 Revised 21 July 2020 Accepted 13 August 2020

Check for updates

(C) Author(s) (or their employer(s)) 2020. Re-use permitted under CC BY-NC. No commercial re-use. See rights and permissions. Published by BMJ.

For numbered affiliations see end of article.

Correspondence to Linda M Delahanty; Delahanty.linda@mgh.harvard. edu and Dr Deborah J Wexler; DWEXLER@mgh.harvard.edu

\section{ABSTRACT}

Introduction We aimed to test the effectiveness of a lifestyle intervention (LI) for individuals with food insecurity and type 2 diabetes.

Research design and methods Adults with type 2 diabetes, body mass index $\geq 25 \mathrm{~kg} / \mathrm{m}^{2}$ (or $\geq 23 \mathrm{~kg} / \mathrm{m}^{2}$ if Asian), hemoglobin A1c of $6.5 \%-11.5 \%(48-97 \mathrm{mmol} / \mathrm{mol})$ and who were willing to lose $5 \%-7 \%$ bodyweight were enrolled in REAL HEALTH-Diabetes. This practice-based randomized clinical trial compared LI (delivered inperson or by telephone) with medical nutrition therapy (MNT) on weight loss at 6 and 12 months. Two or more affirmative responses on the six-item US Department of Agriculture Food Security Survey Module indicated food insecurity. In this prespecified subgroup analysis, we tested using linear mixed effects models whether the intervention effect varied by food security status.

Results of 208 participants, $13 \%$ were food insecure. Those with food insecurity were more likely to be racial/ ethnic minorities $(p<0.001)$ and have lower education $(p<0.001)$. LI, versus MNT, led to greater weight loss at 6 months (5.1\% lost vs $1.1 \%$ lost; $p<0.0001)$ and 12 months (4.7\% lost vs $2.0 \%$ lost; $p=0.0005)$. The intervention effect was similar regardless of food security status $(5.1 \%$ bodyweight lost vs $1.1 \%$ in food secure participants and $5.1 \%$ bodyweight lost vs $1.3 \%$ in food insecure participants at 6 months; $4.7 \%$ bodyweight lost vs $2.1 \%$ in food secure participants and $4.5 \%$ bodyweight lost vs $0.9 \%$ in food insecure participants at 12 months; $p$ for interaction $=0.99$ ).

Conclusions The REAL HEALTH-Diabetes lifestyle intervention led to meaningful weight loss for individuals with food insecurity and type 2 diabetes.

Trial registration number NCT02320253.

The primary goal of type 2 diabetes management is to prevent complications of diabetes by controlling risk factors. ${ }^{1}$ However, the burden of diabetes and its complications does not fall evenly-individuals with lower socioeconomic status face increased risk of diabetes as well as worse diabetes outcomes. ${ }^{2}$ One potentially modifiable contributor to this

\section{Significance of this study}

What is already known about this subject?

- Individuals with diabetes and food insecurity face worse outcomes.

- It is unclear whether lifestyle interventions recommended for diabetes management will be effective for those who experience food insecurity.

What are the new findings?

- A pragmatic lifestyle intervention program led to clinically meaningful weight loss for both food secure and food insecure individuals with type 2 diabetes.

- Weight loss was almost identical for both food insecure and food secure intervention participants.

How might these results change the focus of research or clinical practice?

- Clinicians caring for food insecure individuals with diabetes can recommend lifestyle intervention for weight loss even if they do not have interventions to address food insecurity directly.

is food insecurity, defined as limited access to sufficient food for a healthy, active life. ${ }^{3}$ Food insecurity is strongly associated with greater obesity and worse risk factor control in individuals with diabetes. ${ }^{4-9}$

Typical food insecurity interventions focus on increasing healthy food access by subsidizing or providing healthy food. ${ }^{10}{ }^{11}$ While important, the clinical impact of these approaches is not yet known, and a prior randomized trial of a food pantry-based intervention did not result in changes in diabetes outcomes. ${ }^{12}$ Given the complexity of the relationship between food insecurity and clinical outcomes, ${ }^{5} 13$ additional interventions for individuals with food insecurity which could either be combined with food access interventions or serve as stand-alone approaches 
are needed. Lifestyle intervention (LI), which combines education and skill-building in order to facilitate diabetes self-management, could be one way to meet this need. In both 'efficacy' trials and in more 'real-world' applications, LIs have led to weight loss and improvements in diabetes control, risk factors for diabetes complications, and complications themselves. ${ }^{14-16}$ Further, among food insecure populations, education and skill-building approaches that focus on making best use of available resources, exemplified by Supplemental Nutrition Assistance Program Education (SNAP-Ed), ${ }^{17}$ a program that helps SNAP recipients and communities use farmers markets to address food insecurity, have been shown to be successful in improving diet quality.

Despite the promise of LI, however, few studies have examined LI for management of type 2 diabetes in individuals with food insecurity. Testing the effectiveness of LI in those with food insecurity is important, since food insecurity, for several reasons, is likely to present a barrier to weight loss that may render LI less effective. Mechanistically, food insecurity may act to thwart lifestyle change by incentivizing the consumption of highly processed, calorie-dense foods that are cheaper on a percalorie basis than healthier alternatives. ${ }^{518}$ Further, food insecurity may impose competing demands and increase stress, which makes enacting behavior change more difficult. ${ }^{192}$ Finally, the time commitment required for LI may be prohibitively high for those with many competing demands, such as work and childcare. Alternatively, the skills taught during LI may help facilitate more effective diabetes self-management even in the face of challenging circumstances like food insecurity, if those interventions are appropriately adapted to the needs of individuals with lower socioeconomic status. For these reasons, we believe it is important to determine whether the effectiveness of LI varies by food security status.

In this prespecified subgroup analysis of the REAL HEALTH-Diabetes pragmatic randomized clinical trial, we sought to determine whether an evidence-based LI, delivered in English or Spanish, inperson or via telephone, would result in clinically meaningful weight loss in individuals with type 2 diabetes both with and without food insecurity, and whether the magnitude of weight loss would vary by food security status.

\section{RESEARCH DESIGN AND METHODS}

Participants and study setting

A description of the REAL HEALTH-Diabetes randomized clinical trial design and participants has been previously published. ${ }^{21}{ }^{22}$ In brief, individuals were eligible if they were adults (age $\geq 18$ years) with type 2 diabetes, body mass index $\geq 25 \mathrm{~kg} / \mathrm{m}^{2}$ (or $\geq 23 \mathrm{~kg} / \mathrm{m}^{2}$ if of selfreported Asian ancestry), had hemoglobin A1c (HbAlc) of $6.5 \%-11.5 \%$ (48-97 $\mathrm{mmol} / \mathrm{mol}$ ), had blood pressure $<160 / 100 \mathrm{~mm} \mathrm{Hg}$, and were willing to lose $5 \%-7 \%$ bodyweight. Individuals were drawn from those with a medical provider in the Partners HealthCare clinical network.
Individuals were excluded if they weighed more than $159 \mathrm{~kg}$, spoke a language other than English or Spanish, were pregnant or planning to become pregnant, were currently enrolled in a weight loss program or medical nutrition therapy (MNT), or had a previous or planned bariatric procedure. Further, those who could not participate in the intervention owing to a medical condition that would interfere with the ability to engage in the LI or were taking medications likely to affect weight (eg, corticosteroids) were also excluded. Intervention and data collection visits were conducted either at one of three community health centers or at the Massachusetts General Hospital Diabetes Research Center, depending on the location of the patient's residence.

Participants were recruited between January 2015 and July 2017, with 1-year outcomes analyzed from December 2018 to January 2020. All trial participants provided written informed consent. Prior to participant enrollment, the trial was registered at ClinicalTrials.gov.

\section{Intervention}

REAL HEALTH-Diabetes randomized individuals in a 1:1:1 ratio to inperson group LI, telephone conference call group LI, or MNT. Randomization was accomplished using a computer-generated variable block size randomization scheme stratified by gender and clinical site. The LI was a complex behavioral weight loss intervention developed as a curated hybrid of key publicly available Look AHEAD $^{14}$ and Diabetes Prevention Program (DPP) ${ }^{23}$ session materials and included the first 19 sessions from the original Look AHEAD LI. The LI addressed a variety of nutrition, activity, and behavioral topics and focused on building skills for self-monitoring, goal setting, stimulus control, stress management, problem solving, and relapse prevention. The session materials were culturally sensitive and had a Flesch Reading Ease score of 74.0 and a Flesch-Kincaid grade level of 5.3. However, the materials were not specifically adapted to address the needs of those with food insecurity or low socioeconomic status. Similar to Look AHEAD's LI, participants were encouraged to purchase and use meal replacements in the form of shakes, bars or frozen entrees to replace one to two meals per day to promote weight loss. Unlike the original Look AHEAD LI, which was delivered largely in individual sessions supplemented by some group sessions, the REAL HEALTH LI sessions were delivered by registered dietitians in groups of 4-12 (stratified by English or Spanish delivery of materials) and were delivered at a decreasing cadence of 14 weekly sessions followed by 5 biweekly and 6 monthly sessions, for a total of 25 sessions over 1 year. Participants could also elect to take part in up to three individual sessions during the year. Inperson and telephone conference call sessions were identical in number, duration (approximately 60-90 $\mathrm{min}$ ) and content-the only difference between these sessions was the delivery format. Using a 60 min estimate, this means that planned contact for LI participants across 25 planned sessions was 
25 hours. The planned duration of each optional session was $45 \mathrm{~min}$ or 2.25 hours for three sessions.

Individuals randomized to MNT were referred to individual inperson sessions with a dietitian at their health center or another location of their choice as part of usual care (which is the current recommended standard of care). MNT proceeded per usual care, with an initial visit for evaluation with follow-up sessions as determined by the dietitian and the participant. While LI was provided without cost sharing, MNT could require visit copayments depending on the participant's insurance coverage, as is typical in usual care. For MNT sessions, the planned duration was $60 \mathrm{~min}$ for the first session and $30 \mathrm{~min}$ for each additional session. As in usual care, there was no planned number of sessions for MNT, as that is determined in a dialogue between the registered dietitian and the participant. As an example, if an MNT participant attended one initial session and three follow-up sessions over 12 months, that would be 2.5 hours of contact.

\section{Food insecurity}

Food insecurity assessment occurred at the baseline study visit, prior to randomization. Food insecurity was assessed using the six-item US Department of Agriculture Food Security Survey Module with 12-month recall period. ${ }^{24}$ An example item is: 'The food that I bought just didn't last, and I didn't have money to get more. Was that often, sometimes, or never true for you in the last 12 months? ${ }^{24}$ In accordance with standard scoring, those with two or more affirmative responses were classified as food insecure. ${ }^{24}$ Based on prior work examining food insecurity prevalence in individuals with diabetes, including within the clinical network study participants were drawn from, we anticipated food insecurity prevalence of approximately $20 \% .{ }^{8925}$

In addition to categorizing participants by food security status, we also categorized individuals by household income level, using categories of $<\$ 30000$ per year, $\$ 30$ $000-\$ 50000$ per year, $\$ 50000-\$ 100000$ per year, and $\geq \$ 100000$ per year.

\section{Outcomes}

The primary outcome for this study was weight loss, defined as per cent change in weight from baseline. A 3\% decrease in weight from baseline was considered a clinically meaningful change. Weights were obtained using a calibrated scale in light clothing following a standardized protocol. ${ }^{21}$ The trained research assistant measuring the weights was masked to study arm. Another secondary outcome was HbA1c, which was analyzed at a clinical laboratory certified by the NGSP (formerly the 'National Glycohemoglobin Standardization Program'). To reduce the risk of hypoglycemia with LI, the REAL HEALTHDiabetes trial protocol reduced glucose-lowering medication proactively based on self-monitoring of blood glucose results, anticipated reduction in caloric intake, and use of meal replacements to enhance weight loss. ${ }^{21}$ Finally, we examined session attendance.

\section{Covariates and other data collection}

Information relating to sociodemographics, anthropometrics, and laboratory values was obtained during the baseline visit prior to randomization and at follow-up study visits. ${ }^{21}$

\section{Statistical analyses}

All analyses followed the intention-to-treat approach for intervention arm classification. Because prior analyses found that the inperson and telephone-delivered LI produced similar results, ${ }^{22}$ we combined these intervention arms for analysis, comparing those who received either format of LI with those who received MNT. To account for repeated measures within individuals and possible clustering within the study arms in which the LI was delivered, we used three-level mixed effect models with random effects terms for both the individual and intervention group. We examined changes at 6 and 12 months and used interaction terms (food security (or income category) by intervention modality) to examine whether intervention effect varied by food security status or income category. For these analyses, the food insecurity by intervention modality analysis was considered the primary analysis, with other analyses considered exploratory. Nine $(4.3 \%)$ participants did not report income levels and were excluded from income analyses.

We conducted both unadjusted analyses and analyses that adjusted for possible confounders of the relationship between food insecurity (or income) and the outcomes. For the food insecurity models, the adjustment variables were age, gender, race/ethnicity, education, income, primary language, and health insurance. For the income models, we used the same set of adjustment variables minus income. Missing data were not imputed because missingness was less than $5 \%$ for any variable. All analyses were conducted in SAS V.9.4. A two-sided $p$ value of $\leq 0.05$ was considered statistically significant.

\section{RESULTS}

As previously described, 211 individuals enrolled in the trial and $3(1.4 \%)$ withdrew, leaving 208 participants $(98.6 \%)$ who were included in the intention-to-treat analyses. $^{22}$ The mean age of participants was 61.7 (SD: 10.2) years, $55.3 \%$ were women, $76.9 \%$ were of non-Hispanic white race/ethnicity, and $8.2 \%$ spoke Spanish as their primary language (table 1). Thirteen per cent of participants reported food insecurity, with a similar proportion in the two study arms, as expected in a randomized trial. Overall, those who reported food insecurity were more likely to be women and of racial/ethnic minorities, and to have less education, lower income, and public health insurance.

Compared with those receiving MNT, those receiving LI lost a greater percentage of bodyweight at 6 months ( $5.1 \%$ lost vs $1.1 \%$ lost; $\mathrm{p}<0.0001)$ and 12 months $(4.7 \%$ lost vs $2.0 \%$ lost; $\mathrm{p}=0.0005) .{ }^{22}$ The intervention effect in the LI arm was similar regardless of food security status 
Table 1 Participant characteristics by food security status

\begin{tabular}{|c|c|c|c|c|}
\hline & All & Food secure & Food insecure & $\mathbf{P}_{\text {value }}^{*}$ \\
\hline$n$ & 208 & 180 & 28 & \\
\hline Study arm, n (\%) & & & & 1.00 \\
\hline Lifestyle intervention & $142(68.3)$ & $123(68.3)$ & 19 (67.9) & \\
\hline Medical nutrition therapy & $66(31.7)$ & $57(31.7)$ & $9(32.1)$ & \\
\hline Age, mean (SD) & $61.7(10.2)$ & $62.3(9.9)$ & $58.0(11.5)$ & 0.072 \\
\hline Female, n (\%) & $115(55.3)$ & $93(51.7)$ & $22(78.6)$ & 0.008 \\
\hline Race/ethnicity, n (\%) & & & & $<0.0001$ \\
\hline Non-Hispanic white & $160(76.9)$ & $148(82.2)$ & $12(42.9)$ & \\
\hline Non-Hispanic black & $9(4.3)$ & $9(5.0)$ & $0(0.0)$ & \\
\hline Hispanic/Latino & $28(13.5)$ & $14(7.8)$ & $14(50.0)$ & \\
\hline Non-Hispanic Asian & $7(3.4)$ & $5(2.8)$ & $2(7.1)$ & \\
\hline Other & $4(1.9)$ & $4(2.2)$ & $0(0.0)$ & \\
\hline Education, n (\%) & & & & 0.0001 \\
\hline Less than high school diploma & $17(8.2)$ & $10(5.6)$ & $7(25.0)$ & \\
\hline High school diploma or GED & $43(20.7)$ & $39(21.7)$ & $4(14.3)$ & \\
\hline $1-3$ years of college & $60(28.8)$ & $47(26.1)$ & $13(46.4)$ & \\
\hline 4 or more years of college or graduate school & $88(42.3)$ & $84(46.7)$ & $4(14.3)$ & \\
\hline Annual income, $\mathrm{n}(\%)$ & & & & $<0.0001$ \\
\hline$<\$ 30000$ & $48(23.1)$ & $30(16.7)$ & $18(64.3)$ & \\
\hline$\$ 30000-\$ 50000$ & $21(10.1)$ & $19(10.6)$ & $2(7.1)$ & \\
\hline$\$ 50000-\$ 100000$ & $68(32.7)$ & $65(36.1)$ & $3(10.7)$ & \\
\hline$\geq \$ 100000$ & $62(29.8)$ & $60(33.3)$ & $2(7.1)$ & \\
\hline Did not report & $9(4.3)$ & $6(3.3)$ & $3(10.7)$ & \\
\hline Insurance, $\mathrm{n}(\%)$ & & & & 0.001 \\
\hline Commercial & $88(42.3)$ & $80(44.4)$ & $8(28.6)$ & \\
\hline Medicaid & 29 (13.9) & $25(13.9)$ & $4(14.3)$ & \\
\hline Medicare & $59(28.4)$ & $55(30.6)$ & $4(14.3)$ & \\
\hline Medicare and Medicaid ('dual eligible') & $26(12.5)$ & $16(8.9)$ & $10(35.7)$ & \\
\hline Self-pay & $6(2.9)$ & $4(2.2)$ & $2(7.1)$ & \\
\hline Spanish language, n (\%) & $17(8.2)$ & $9(5.0)$ & $8(28.6)$ & 0.0004 \\
\hline $\begin{array}{l}\text { Baseline hemoglobin A1c } \\
\text { NGSP } \% \text {, mean (SD) }\end{array}$ & $7.7(1.2)$ & $7.7(1.2)$ & $8.0(1.2)$ & 0.25 \\
\hline $\mathrm{mmol} / \mathrm{mol}$, mean (SD) & $61.1(12.8)$ & $60.6(12.7)$ & $63.7(13.2)$ & \\
\hline Baseline body mass index, $\mathrm{kg} / \mathrm{m}^{2}$, mean (SD) & $35.0(5.4)$ & $35.0(5.5)$ & $35.3(4.7)$ & 0.75 \\
\hline Baseline weight, kg, mean (SD) & $98.1(18.9)$ & $98.9(19.2)$ & $93.4(16.1)$ & 0.11 \\
\hline Baseline use of any glucose-lowering medication & $191(91.8)$ & $163(90.6)$ & $28(100)$ & 0.14 \\
\hline Baseline use of metformin & $166(79.8)$ & $141(78.3)$ & $25(89.3)$ & 0.21 \\
\hline Baseline use of insulin & $69(33.2)$ & $53(29.4)$ & $16(57.1)$ & 0.005 \\
\hline Baseline use of GLP-1 RA & $14(6.7)$ & $13(7.2)$ & $1(3.6)$ & 0.70 \\
\hline Baseline use of sulfonylurea & 65 (31.3) & $58(32.2)$ & $7(25.0)$ & 0.52 \\
\hline Baseline use of thiazolidinedione & $2(1.0)$ & $2(1.1)$ & $0(0.0)$ & 1.00 \\
\hline Baseline use of DPP-4 & $9(4.3)$ & $8(4.4)$ & $1(3.6)$ & 1.00 \\
\hline Baseline use of SGLT2 & $6(2.9)$ & $4(2.2)$ & $2(7.1)$ & 0.19 \\
\hline
\end{tabular}

${ }^{*} \mathrm{P}$ value from two-sample t-tests for continuous variables and Fisher's exact tests for categorical variables.

DPP-4, dipeptidyl peptidase-4 inhibitor; GED, General Education Development certificate; GLP-1 RA, glucagon-like peptide 1 receptor agonist; NGSP, National Glycohemoglobin Standardization Program; SGLT2, sodium/glucose cotransporter 2 inhibitor. 
Table 2 Per cent change in weight and hemoglobin A1c from baseline at 6 and 12 months

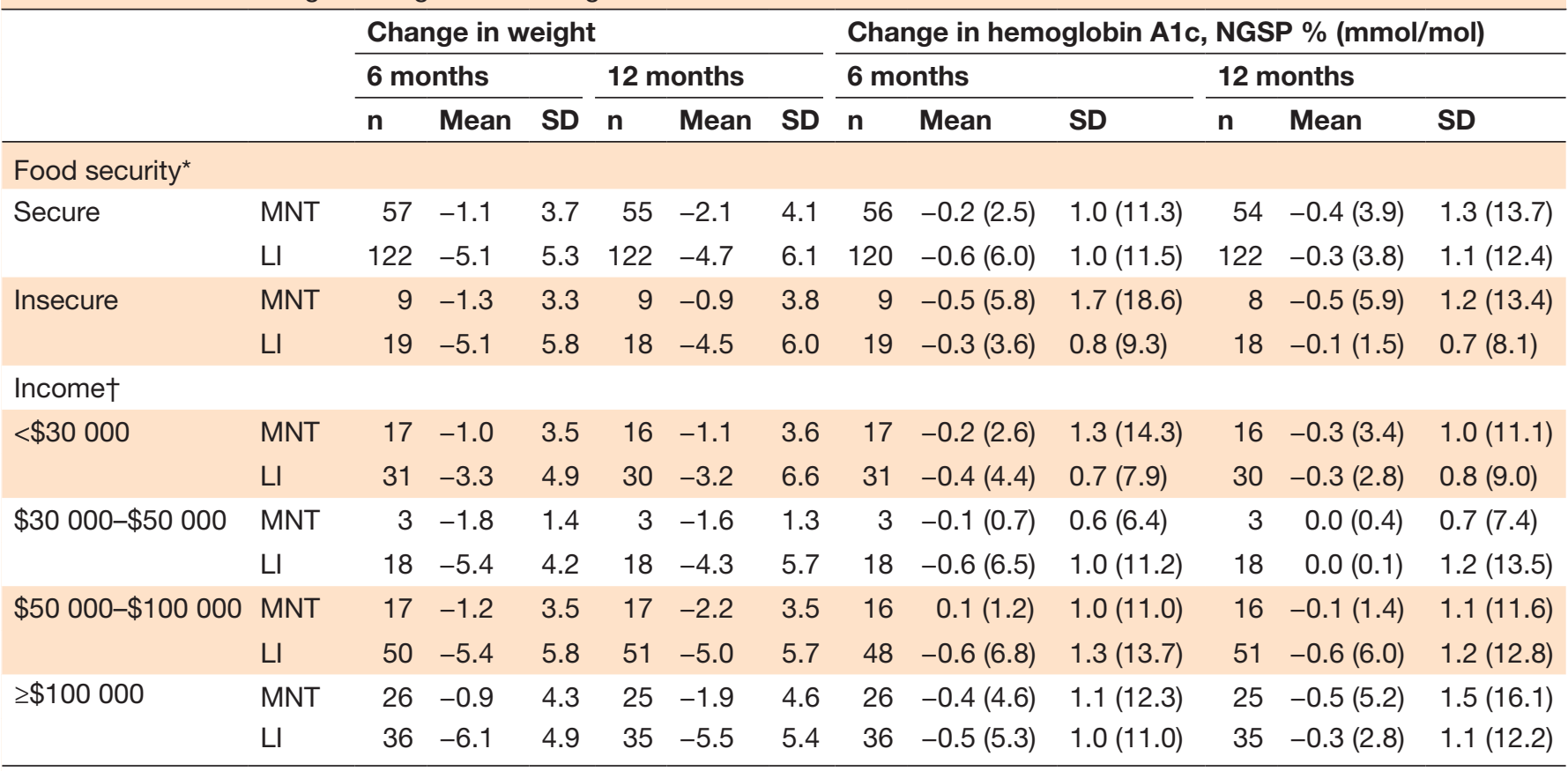

${ }^{*} \mathrm{P}$ value for food security by intervention interaction was 0.99 for weight change and 0.27 for HbA1c change in the model adjusted for age, gender, race/ethnicity, education, primary language, income, and health insurance.

†Participants (4.3\%) who did not report income are excluded from these analyses; $p$ value for income category by intervention interaction was 0.69 for weight change and 0.29 for $\mathrm{HbA} 1 \mathrm{c}$ change in the model adjusted for age, gender, race/ethnicity, education, primary language, and health insurance.

HbA1c, hemoglobin A1c; LI, lifestyle intervention; MNT, medical nutrition therapy; NGSP, National Glycohemoglobin Standardization Program.

( $5.1 \%$ bodyweight lost vs $1.1 \%$ in food secure participants and $5.1 \%$ bodyweight lost vs $1.3 \%$ in food insecure participants at 6 months; $4.7 \%$ bodyweight lost vs $2.1 \%$ in food secure participants and $4.5 \%$ bodyweight lost vs $0.9 \%$ in food insecure participants at 12 months) (table 2, figure 1). Interaction terms testing these differences in intervention effect were not statistically significant in either unadjusted $(\mathrm{p}=0.87)$ or adjusted $(\mathrm{p}=0.99)$ models.

Change in HbAlc did not differ between the LI and MNT groups. HbAlc was $0.5 \%(5.7 \mathrm{mmol} / \mathrm{mol})$ lower

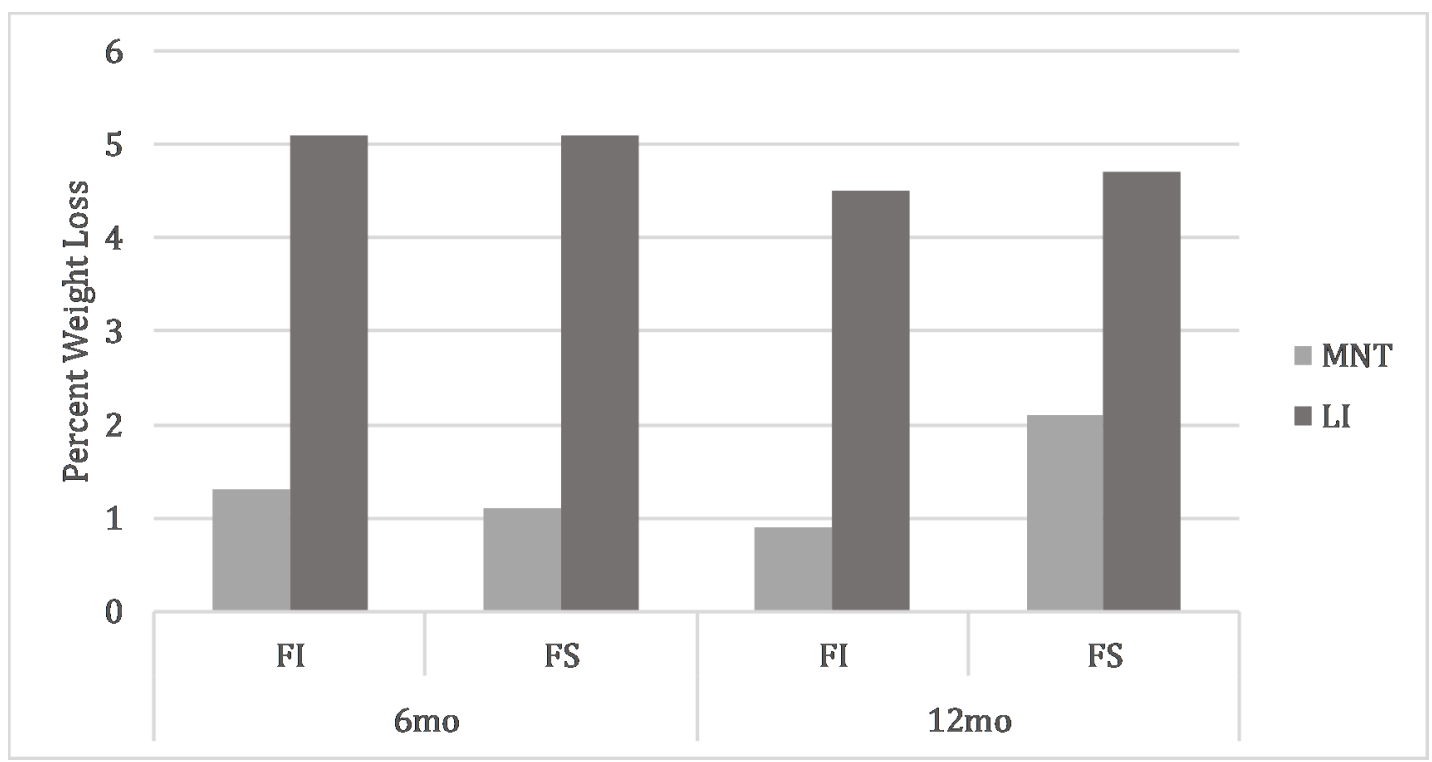

Figure 1 Percentage weight loss at 6 and 12 months by intervention arm (LI, lifestyle intervention; MNT, medically nutrition therapy) among those who are food insecure (FI) and food secure (FS). P value for interaction term testing whether the intervention effect varies among subgroups was 0.87 in unadjusted models and 0.99 in models adjusted for age, gender, race/ ethnicity, education, primary language, income, and health insurance. 


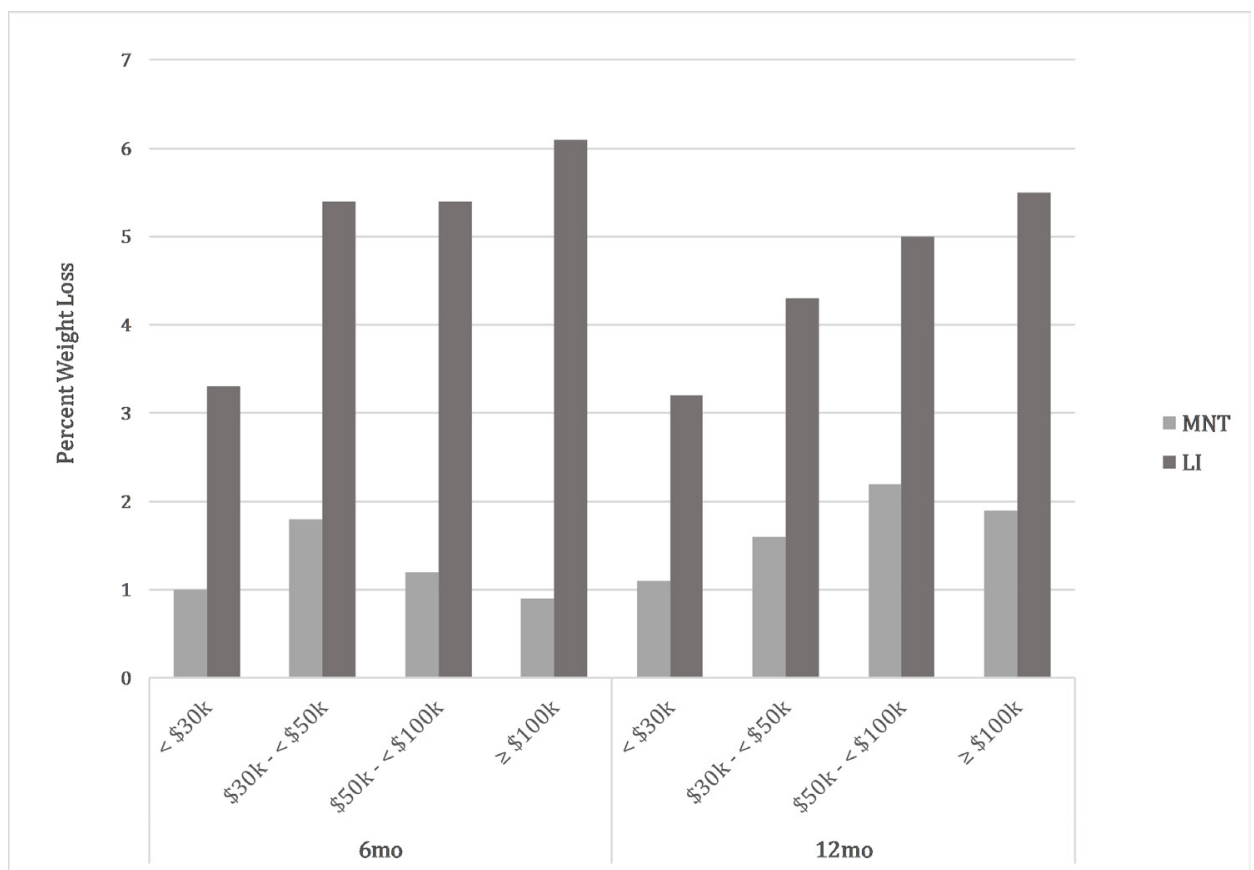

Figure 2 Percentage weight loss at 6 and 12 months by intervention arm (LI, lifestyle intervention; MNT, medical nutrition therapy) by category of annual income ( $\mathrm{k}$ indicates thousands of dollars). $\mathrm{P}$ value for interaction term testing whether the intervention effect varies among subgroups was 0.72 in unadjusted models and 0.69 in models adjusted for age, gender, race/ ethnicity, education, primary language, income, and health insurance.

than baseline in the lifestyle group at 6 months vs $0.3 \%$ $(3.0 \mathrm{mmol} / \mathrm{mol})$ lower in the MNT group $(\mathrm{p}=0.20)$. HbAlc was $0.3 \%(3.5 \mathrm{mmol} / \mathrm{mol})$ lower with LI vs $0.4 \%$ $(4.1 \mathrm{mmol} / \mathrm{mol})$ lower with MNT at 12 months $(\mathrm{p}=0.63)$. In the LI group, change in HbAlc did not vary by food security status. $\mathrm{HbAlc}$ was $0.6 \%(6.0 \mathrm{mmol} / \mathrm{mol})$ lower than baseline in food secure vs $0.3 \%(3.6 \mathrm{mmol} / \mathrm{mol})$ lower in food insecure participants at 6 months. HbAlc was $0.3 \%(3.8 \mathrm{mmol} / \mathrm{mol})$ lower in food secure vs $0.1 \%$ $(1.5 \mathrm{mmol} / \mathrm{mol})$ lower than food insecure at 12 months. The $\mathrm{p}$ values for the difference between food secure and food insecure participants were 0.26 in unadjusted models and 0.28 in adjusted models.

Those with lower income were more likely to be women and of racial/ethnic minorities, to have less education, and to be food insecure (online supplemental eTable 1). The intervention effect on the percentage of bodyweight lost was also similar regardless of income category (table 2, figure 2). The intervention effect was nominally smaller in the lowest income category $(3.3 \%$ lost for LI vs $1.0 \%$ lost for MNT in those with income $<\$ 30000 \mathrm{a}$ year, compared with the overall average of $5.6 \%$ lost vs $1.1 \%$ loss). However, these interactions were not statistically significant in either unadjusted $(\mathrm{p}=0.72)$ or adjusted $(p=0.68)$ models. The intervention effect on HbAlc also did not significantly differ by income category (table 2 ).

Examining differences in attendance in LI sessions (online supplemental eTable 2), those who were food insecure attended approximately three fewer intervention sessions at both 6 and 12 months compared with food secure individuals, but this difference was not statistically significant at $6(\mathrm{p}=0.06)$ or $12(\mathrm{p}=0.17)$ months.
The average attendance in LI in the food secure group was 14.8 out of 19 sessions in the first 6 months (14.8 hours of contact received relative to 19 hours planned) and 18.6 sessions out of 25 planned (18.6 hours received relative to 25 hours planned) at 12 months. For food insecure LI participants, the average attendance was 11.8 out of 19 sessions ( 11.8 hours received relative to 19 hours planned) in the first 6 months and 15.9 out of 25 sessions at 12 months (15.9 hours received relative to 25 hours planned). Optional sessions attended are described in online supplemental eTable 3. In the MNT arm, those with food insecurity attended 1.6 fewer visits $(p=0.009)$ at 6 months and 2.9 fewer visits $(p=0.0002)$ at 12 months than food secure participants. For food secure MNT participants, the mean attendance was 2.5 sessions (1.75 hours received) at 6 months and 4.0 sessions (2.5 hours received) at 12 months. For food insecure MNT participants, visit attendance was 0.9 sessions (0.9 hours received) at 6 months and 1.1 sessions (1.05 hours received) at 12 months. Visit attendance did not vary significantly by income in either arm (online supplemental eTable 2).

\section{DISCUSSION}

In this preplanned subgroup analysis of a pragmatic randomized trial, we found that the LI led to clinically meaningful weight loss in food insecure individuals. Further, although there were small variations in per cent weight lost by income category, the differences across groups were neither statistically significant nor clinically meaningful. There was no evidence that factors that 
may confound the relationship between food insecurity (or low income) and weight loss (eg, race/ethnicity or education) affected the results observed.

The major contribution of this study is to examine whether the effect of an LI for weight loss varied by food security status in individuals with type 2 diabetes. To our knowledge, whether an LI is similarly effective in those with, versus without, food insecurity has not previously been tested. However, there have been several prior studies addressing weight loss among low-income individuals with type 2 diabetes. A randomized controlled trial (RCT) that combined lifestyle and psychosocial intervention strategies in a low-income sample with diabetes found only modest weight loss $(1.2 \mathrm{~kg}$ difference compared with usual care). ${ }^{26} \mathrm{~A}$ community-based diabetes self-management intervention for low-income African-American participants did not find changes in weight. ${ }^{27}$ This study did report improvements in HbAlc at 6 months, but these were not sustained at 12 or 18 months. A community health worker-led intervention for African-American women, primarily with lower income, found a modest difference in weight lost $(1.35 \mathrm{~kg}$ lost vs $0.39 \mathrm{~kg}$, respectively; $\mathrm{p}=0.046$ ), using an educational mailings comparison group. ${ }^{28}$ A pilot RCT for AfricanAmerican individuals with diabetes that used dietitian-led counseling sessions with weekly peer-support telephone calls, in a sample where $72 \%$ had an annual household income $<\$ 20000$, did not find a significant effect on weight loss. ${ }^{29}$ None of the studies mentioned examined whether intervention effect varied by food security status. Further, studies that are restricted to individuals with lower income are unable to determine whether intervention effects vary in lower, compared with higher, income groups.

Two other studies that did not focus on weight loss but did examine food insecurity are worth a discussion. A previous study of a diabetes self-management intervention, which included dietary advice but was not focused on weight loss, did find that food insecure participants showed similar (or larger) improvements in diabetes management self-efficacy and HbAlc, compared with food secure participants. ${ }^{30}$ A previous randomized trial of a food pantry-based intervention providing healthy food and diabetes management support for food insecure individuals found improvements in fruit and vegetable intake, but no change in HbA1c or weight. ${ }^{12}$

In the REAL HEALTH trial, because of anticipated weight loss which could lead to hypoglycemia if glucoselowering medications were not adjusted, trial protocols emphasized reduction of glucose-lowering medications when appropriate. ${ }^{21}$ Thus the lack of difference in glycemic control between MNT and LI was anticipated. For the purpose of this subgroup analysis, the key finding is that similar HbA1c reductions were observed regardless of food security status (or income category).

An important observation in this study is the lower than anticipated prevalence of food insecurity in the included sample. Prior estimates of the patient population from which the study sample was drawn, including a study in the same clinical network, indicate a food insecurity prevalence of greater than $20 \%,{ }^{89}$ while only $13 \%$ of REAL HEALTH-Diabetes participants were food insecure. This suggests that there may be barriers to enrollment in LI among groups who experience food insecurity, including the length of the program and other competing demands, along with food insecurity itself. Food insecure individuals also attended approximately three fewer LI sessions, although this difference was not statistically significant, and attended significantly fewer MNT sessions. The benefits seen in this study, in which participants did not incur out-of-pocket costs for LI, suggest that if LI is more widely implemented it should be offered without cost-sharing requirements, as these are likely only to reduce participation, further increase the financial burden of diabetes, and lead to cost-related non-adherence and deterioration of glycemic control. ${ }^{31}$ Future work may address this barrier by combining interventions. For example, an intervention might combine directly addressing food insecurity (via food or medically tailored meal provision) with LI. By providing nutrition support, such a combined intervention may make the benefits of LI available to individuals who may otherwise not feel able to commit to lifestyle change. At the same time, the skills learned during LI could help sustain dietary modification as individuals transition off of food support interventions.

The findings of this study have important implications for diabetes management among vulnerable patients. Because this LI was effective regardless of food security status or income category, it may be offered in clinics, such as health centers, that serve populations with low income and/or high food insecurity prevalence. Because LI is an effective alternative to MNT (which is often a benefit covered by health insurance), state Medicaid programs and private health insurers should consider adding LIs as covered benefits-much as Medicare has recently done in allowing coverage for DPP-derived services in certain contexts. ${ }^{32}$ Given the ease of administration of the telephonic intervention, such an approach may be a particularly attractive strategy among individuals with transportation barriers or schedules that preclude inperson sessions.

The results of this study should be interpreted in light of several limitations. Although this was a prespecified subgroup analysis, the study did not randomize on the basis of food insecurity or income. Nevertheless, since treatment assignment was made without regard to food security status, food security status cannot confound the comparisons made between the LI and MNT group, within levels of food security status. Further, adjustment for known confounders did not change the finding of no effect modification. ${ }^{33}$ Next, the LI was offered to participants without cost sharing, whereas MNT was not covered as part of the study, and therefore copays and deductibles could have precluded some food insecure participants from receiving the recommended efficacious dose of MNT. ${ }^{34}$ However, this is unlikely to have affected 
the study results as food secure individuals, who should face fewer financial barriers to MNT, saw weight loss in the MNT arm that was similar to that seen in food insecure participants. Next, as noted above, the prevalence of food insecurity was lower in the study sample than would be expected in the background population. Given the effect estimates were not greater than a prespecified clinically meaningful threshold across the subgroups, the observation of similar effects regardless of food insecurity status (or income level) is not likely to be affected by low power, but this type of selection into the trial may reduce the generalizability of the results. Specifically, those who, owing to food insecurity, felt incapable of committing to an LI and thus did not enroll may have experienced less intervention benefit if they had enrolled. Next, we do not present information on possible mediators of intervention effect, such as gain of knowledge or changes in attitudes or behaviors. That will be an important direction for future work. Another limitation is that, owing to the small sample of food insecure individuals, we did not have sufficient power to stratify analyses by LI modality (telephone vs inperson). Finally, this study was conducted in a single clinical network in eastern Massachusetts; thus, results may not be generalizable throughout the USA. The limitations are balanced by several strengths. This was a pragmatic but rigorous randomized trial of an LI adapted for group implementation in primary care and community health centers. The intervention was conducted in English and Spanish, and the subgroup analyses reported here were prespecified.

We found that an LI was effective for weight loss in individuals with type 2 diabetes and food insecurity and/ or low income who enrolled in the program. However, barriers to enrolling in LI remain a concern. New research that combines the ability to support those experiencing food insecurity in making lifestyle changes that modify their disease trajectory is an important next step that may help us to reduce socioeconomic disparities in diabetes.

\section{Author affiliations \\ ${ }^{1}$ Division of General Medicine \& Clinical Epidemiology, Department of Medicine, University of North Carolina at Chapel Hill School of Medicine, Chapel Hill, North Carolina, USA \\ ${ }^{2}$ Department of Medicine, Harvard Medical School, Boston, Massachusetts, USA ${ }^{3}$ Division of General Internal Medicine, Massachusetts General Hospital, Boston, Massachusetts, USA \\ ${ }^{4}$ Diabetes Unit, Massachusetts General Hospital, Boston, Massachusetts, USA}

Acknowledgements The authors thank the participants of the study and the REAL HEALTH-Diabetes study team: Douglas E Levy PhD, Valerie Goldman MS RD, Jeanna McCarthy MS RD, Laurie Bissett MS RD, Anthony Romeo Rodriguez BS, Barbara Chase ANP APRN, Rajani LaRocca MD, Amy Wheeler MD, and Amy Dushkin BS. Finally, the authors gratefully acknowledge consultation from the New York Regional Center for Diabetes Translation Research (P30 DK111022) on the design and implementation of the clinical trial.

Contributors SAB, DJW, and LMD conceived of the study and drafted the manuscript. YC, SJC, and BP assisted with analysis and interpretation of the data and revised the manuscript critically for intellectual content. All authors gave approval of the manuscript version to be submitted. SAB, LMD and DJW are guarantors and had full access to the data and take responsibility for the work as a whole, including study design, data integrity and accuracy of the analysis.

Funding Funding for this study was provided by the National Institute of Diabetes and Digestive and Kidney Diseases of the National Institutes of Health under Award Number R18DK102737, K23DK109200, and T32DK007028. The content is solely the responsibility of the authors and does not necessarily represent the official views of the National Institutes of Health.

Competing interests DJW reports serving on a data monitoring committee for Novo Nordisk. LMD serves on the advisory boards for Omada Health, Jana Care, and WW International. SJC reports employment of a close family member by Johnson \& Johnson.

\section{Patient consent for publication Not required.}

Ethics approval The trial was approved by the institutional review board at Partners HealthCare (protocol number 2014P002074).

Provenance and peer review Not commissioned; externally peer reviewed.

Data availability statement Data are available upon reasonable request.

Open access This is an open access article distributed in accordance with the Creative Commons Attribution Non Commercial (CC BY-NC 4.0) license, which permits others to distribute, remix, adapt, build upon this work non-commercially, and license their derivative works on different terms, provided the original work is properly cited, appropriate credit is given, any changes made indicated, and the use is non-commercial. See: http://creativecommons.org/licenses/by-nc/4.0/.

\section{ORCID ID}

Linda M Delahanty http://orcid.org/0000-0002-1525-3559

\section{REFERENCES}

1 American Diabetes Association. Standards of medical care in Diabetes-2019. Diabetes Care 2019;42:S1-2.

2 American Diabetes Association. 1. promoting health and reducing disparities in populations. Diabetes Care 2017;40:S6-10.

3 Coleman-Jensen A, Rabbitt MP, Gregory CA, et al. Household food security in the United States in 2017. Available: https://www.ers. usda.gov/publications/pub-details/?pubid=90022 [Accessed 25 Sep 2018]

4 Morales ME, Berkowitz SA. The relationship between food insecurity dietary patterns, and obesity. Curr Nutr Rep 2016;5:54-60.

5 Seligman HK, Schillinger D. Hunger and socioeconomic disparities in chronic disease. N Engl J Med 2010;363:6-9.

6 Seligman HK, Davis TC, Schillinger D, et al. Food insecurity is associated with hypoglycemia and poor diabetes self-management in a low-income sample with diabetes. J Health Care Poor Underserved 2010;21:1227-33.

7 Seligman HK, Jacobs EA, López A, et al. Food insecurity and glycemic control among low-income patients with type 2 diabetes. Diabetes Care 2012;35:233-8.

8 Berkowitz SA, Meigs JB, DeWalt D, et al. Material need insecurities, control of diabetes mellitus, and use of health care resources: results of the measuring economic insecurity in diabetes study. JAMA Intern Med 2015;175:257-65.

9 Berkowitz SA, Baggett TP, Wexler DJ, et al. Food insecurity and metabolic control among U.S. adults with diabetes. Diabetes Care 2013;36:3093-9.

10 De Marchis EH, Torres JM, Benesch T, et al. Interventions addressing food insecurity in health care settings: a systematic review. Ann Fam Med 2019;17:436-47.

11 Hessler D, Bowyer V, Gold R, et al. Bringing social context into diabetes care: intervening on social risks versus providing Contextualized care. Curr Diab Rep 2019;19:30.

12 Seligman HK, Smith M, Rosenmoss S, et al. Comprehensive diabetes self-management support from food banks: a randomized controlled trial. Am J Public Health 2018;108:1227-34.

13 National Academies of Sciences, Engineering, and Medicine. Integrating Social Care into the Delivery of Health Care: Moving Upstream to Improve the Nation's Health. The National Academies Press, 2019.

14 Look AHEAD Research Group, Wing RR. Long-Term effects of a lifestyle intervention on weight and cardiovascular risk factors in individuals with type 2 diabetes mellitus: four-year results of the look ahead trial. Arch Intern Med 2010;170:1566-75.

15 Evert AB, Dennison M, Gardner CD, et al. Nutrition therapy for adults with diabetes or prediabetes: a consensus report. Diabetes Care 2019;42:731-54. 
16 Huang X-L, Pan J-H, Chen D, et al. Efficacy of lifestyle interventions in patients with type 2 diabetes: a systematic review and metaanalysis. Eur J Intern Med 2016;27:37-47.

17 Rivera RL, Maulding MK, Eicher-Miller HA. Effect of supplemental nutrition assistance Program-Education (SNAP-Ed) on food security and dietary outcomes. Nutr Rev 2019;77:903-21.

18 Seligman HK, Berkowitz SA. Aligning programs and policies to support food security and public health goals in the United States. Annu Rev Public Health 2019;40:319-37.

19 Ippolito MM, Lyles CR, Prendergast K, et al. Food insecurity and diabetes self-management among food pantry clients. Public Health Nutr 2017;20:183-9.

20 Berkowitz SA, Seligman HK, Choudhry NK. Treat or eat: food insecurity, cost-related medication underuse, and unmet needs. Am J Med 2014;127:303-10.

21 Delahanty LM, Chang Y, Levy DE, et al. Design and participant characteristics of a primary care adaptation of the look ahead lifestyle intervention for weight loss in type 2 diabetes: the real HEALTH-diabetes study. Contemp Clin Trials 2018;71:9-17.

22 Delahanty LM, Levy DE, Chang Y, et al. Effectiveness of lifestyle intervention for type 2 diabetes in primary care: the real HEALTHDiabetes randomized clinical trial. J Gen Intern Med 2020;359.

23 Diabetes Prevention Program (DPP) Research Group. The diabetes prevention program (DPP): description of lifestyle intervention. Diabetes Care 2002;25:2165-71.

24 United States Department of Agriculture Economic Research Service. Food security survey module, 2018. Available: https://www. ers.usda.gov/topics/food-nutrition-assistance/food-security-in-theus/survey-tools/

25 Berkowitz SA, Berkowitz TSZ, Meigs JB, et al. Trends in food insecurity for adults with cardiometabolic disease in the United States: 2005-2012. PLoS One 2017;12:e0179172.

26 Moncrieft AE, Llabre MM, McCalla JR, et al. Effects of a multicomponent life-style intervention on weight, glycemic control, depressive symptoms, and renal function in low-income, minority patients with type 2 diabetes: results of the community approach to lifestyle modification for diabetes randomized controlled trial. Psychosom Med 2016;78:851-60.

27 Lynch EB, Mack L, Avery E, et al. Randomized trial of a lifestyle intervention for urban low-income African Americans with type 2 diabetes. J Gen Intern Med 2019;34:1174-83.

28 Lutes LD, Cummings DM, Littlewood K, et al. A community health Worker-Delivered intervention in African American women with type 2 diabetes: a 12-month randomized trial. Obesity 2017;25:1329-35.

29 Lynch EB, Liebman R, Ventrelle J, et al. A self-management intervention for African Americans with comorbid diabetes and hypertension: a pilot randomized controlled trial. Prev Chronic Dis 2014;11:E90.

30 Lyles CR, Wolf MS, Schillinger D, et al. Food insecurity in relation to changes in hemoglobin A1c, self-efficacy, and fruit/vegetable intake during a diabetes educational intervention. Diabetes Care 2013;36:1448-53.

31 Patel MR, Resnicow K, Lang I, et al. Solutions to address diabetesrelated financial burden and Cost-Related nonadherence: results from a pilot study. Health Educ Behav 2018;45:101-11.

32 Centers for Medicare \& Medicaid Services (CMS), HHS. Medicare program; revisions to payment policies under the physician fee schedule and other revisions to part B for cy 2018; Medicare shared savings program requirements; and Medicare diabetes prevention program. final rule. Fed Regist 2017;82:52976-3371.

33 VanderWeele TJ, Knol MJ. Interpretation of subgroup analyses in randomized trials: heterogeneity versus secondary interventions. Ann Intern Med 2011;154:680-3.

34 Franz MJ, MacLeod J, Evert A, et al. Academy of nutrition and dietetics nutrition practice guideline for type 1 and type 2 diabetes in adults: systematic review of evidence for medical nutrition therapy effectiveness and recommendations for integration into the nutrition care process. J Acad Nutr Diet 2017;117:1659-79. 